\title{
The Role of Lung Ultrasound in Diagnosis of Respiratory Distress Syndrome in Newborn Infants
}

\author{
Jing Liu ${ }^{1}$; Hai Ying Cao ${ }^{2, *}$; Hua-Wei Wang ${ }^{1}$; Xiang Yong Kong ${ }^{1}$ \\ ${ }_{1}^{1}$ Department of Neonatology and NICU, Bayi Children's Hospital Affiliated to Beijing Military General Hospital, Beijing, China \\ ${ }^{2}$ Department of Ultrasonography, General Electric (GE) Healthcare of USA, Beijing, China \\ ${ }^{*}$ Corresponding author: Hai Ying Cao, Department of Neonatology and NICU, Bayi Children's Hospital Affiliated to Beijing Military General Hospital, 5 Nanmen Cang Dongcheng \\ District Beijing 100700, Beijing, China. E-mail: liujingbj@live.cn \\ Received: June 25, 2013; Accepted: January 12, 2014
}

\begin{abstract}
Background: Respiratory distress syndrome (RDS) is one of the most common causes of neonatal respiratory failure and mortality. The risk of developing RDS decreases with both increasing gestational age and birth weight.

Objectives: The aim of this study was to evaluate the value of lung ultrasound in the diagnosis of respiratory distress syndrome (RDS) in newborn infants.

Materials and Methods: From March 2012 to May 2013, 100 newborn infants were divided into two groups: RDS group (50 cases) and control group (50 cases). According to the findings of chest x-ray, there were 10 cases of grade II RDS, 15 grade III cases, and 25 grade IV cases in RDS group. Lung ultrasound was performed at bedside by a single expert. The ultrasound indexes observed in this study included pleural line, A-line, B-line, lung consolidation, air bronchograms, bilateral white lung, interstitial syndrome, lung sliding, lung pulse etc. Results: In all of the infants with RDS, lung ultrasound consistently showed generalized consolidation with air bronchograms, bilateral white lung or alveolar-interstitial syndrome, pleural line abnormalities, A-line disappearance, pleural effusion, lung pulse, etc. The simultaneous demonstration of lung consolidation, pleural line abnormalities and bilateral white lung, or lung consolidation, pleural line abnormalities and A-line disappearance co-exists with a sensitivity and specificity of $100 \%$. Besides, the sensitivity was $80 \%$ and specificity $100 \%$ of lung pulse for the diagnosis of neonatal RDS.

Conclusions: This study indicates that using an ultrasound to diagnose neonatal RDS is accurate and reliable too. A lung ultrasound has many advantages over other techniques. Ultrasound is non-ionizing, low-cost, easy to operate, and can be performed at bedside, making this technique ideal for use in NICU.

Keywords: Lung; Respiratory Distress Syndrome, Newborn; Infant, Newborn
\end{abstract}

\section{Background}

Respiratory distress syndrome (RDS) is one of the most common causes of neonatal respiratory failure and neonatal mortality. The underlying pathogenesis of the disease involves developmental immaturity of the lungs, leading to the absence of pulmonary surfactant. The risk of developing RDS decreases with both increasing gestational age and birth weight. The incidence rate is $80 \%$ in infants $<28$ weeks' gestation, $60 \%$ at 29 weeks, $15-30 \%$ at 32-34 weeks, and declines with maturity to 5\% at 35-36 weeks. Accordingly, the RDS incidence rate is estimated to be $80 \%$ for infants weighing $<750 \mathrm{~g}$ at birth and $55 \%$ for infants weighing 750-1000 g (1). However, in recent years, with the application of antenatal corticosteroids and delivery room pulmonary surfactant, both typical and severe RDS in premature infants have greatly declined. Greater awareness of RDS has led to a more frequent diagnosis in term neonates (2-4). Bouziri et al. (3) found that RDS accounted for $6.8 \%$ of cases of respiratory distress in term or near-term infants. We have also found that RDS in term infants accounts for 3.6\% of all term in-patients (5). The diagnosis of RDS is usually based on clinical manifes- tations, arterial blood gas analysis and chest x-rays. Lung ultrasound is typically not included in the diagnostic work-up of neonatal RDS. Recently, ultrasound has been used extensively and successfully in the diagnosis of many kinds of lung diseases (6-13) and a few studies have addressed neonatal RDS $(14,15)$.

\section{Objectives}

This study aims to further evaluate the value of a lung ultrasound in the diagnosis of RDS in newborn infants including full-term neonates.

\section{Materials and Methods}

\subsection{Patients}

This study was approved by the ethics committee of the General Hospital of Beijing Military Command. From March 2012 to May 2013, 50 newborn infants with RDS and another 50 neonates whose lung disease was excluded by chest x-ray examination were enrolled in this study, all of

Copyright (C) 2015, Growth \& Development Research Center. This is an open-access article distributed under the terms of the Creative Commons Attribution-NonCommercial 4.0 International License (http://creativecommons.org/licenses/by-nc/4.0/) which permits copy and redistribute the material just in noncommercial usages, provided the original work is properly cited. 
whom were admitted to the Department of Neonatology and NICU (the largest NICU in the world with 350 beds), Bayi Children's Hospital affiliated to Beijing Military General Hospital (Beijing, China). Beside the premature infants, the diagnostic criteria of RDS in full-term neonates were as follow (2-5):1) full-term neonates (gestational age $\geq 37$ weeks), 2) acute onset, 3) an acute, explicit perinatal triggering insult such as severe perinatal acquired infection, severe birth asphyxia, meconium aspiration syndrome, or delivery by selective cesarean section, etc., 4) representative clinical manifestations including progressive respiratory distress occurring shortly after birth, tachypnea, expiratory grunting, nasal flaring, subcostal retractions, cyanosis, reduced or absent breath sounds, or severe dyspnea requiring continuous positive pressure ventilation support for at least 72 hours, 5) typical chest $\mathrm{x}$-ray abnormalities such as hypoexpansion, diffuse, fine granular densities, air bronchogram signs, ground-glass opacities, blurred cardiac borders, or white lungs (whiteout appearance), 6) arterial blood gas analysis showing hypoxia, hypercapnia and an oxygen tension/fraction of inspired oxygen ratio $\left(\mathrm{PaO}_{2} / \mathrm{FiO}_{2}\right) \leq 26.7 \mathrm{kPa}$. Those patients, whose dyspnea was caused only by severe infection, meconium aspiration syndrome, etc., were excluded.

\subsection{Methods}

This was a double blind study. A high-resolution line probe with a frequency of more than $7.5 \mathrm{MHz}$ (generally 11-12 MHz) (GE Voluson i or E6, USA) was used in this study. A lung ultrasound was performed at bedside by a single expert immediately after admission. The transthoracic approach was performed with longitudinal scans of the anterior and posterior chest walls. The ultrasound execution cut-off time was 5 minutes. A conventional anteroposterior chest $\mathrm{x}$-ray was performed at the bedside on RDS patients immediately following the lung ultrasound, and was read by an independent radiologist who was unaware of the ultrasound results.

\subsection{Ultrasound Indexes Used in This Study}

1) Pleural line: the regular echogenic line under the superficial layers of the thorax moving continuously during respiration, while abnormal pleural lines refer to pleural lines disappearance, indistinct or thicker in width more than $0.5 \mathrm{~cm}$ (16). 2) A-line: a series of echogenic, horizontal, parallel lines equidistant from one another below the pleural line, which are the reverberation artifacts of the pleural line (17, 18). 3) B-lines: also known as ultrasound lung comets, hyperechoic narrow-based artifacts spreading like laser rays from the pleural line to the edge of the screen (19). 4) Lung consolidation: defined as areas of hepatization (tissue pattern) with presence of air bronchograms or fluid bronchograms $(18,20) .5)$ Pleural effusion: defined as anechoic-dependent collections limited by the diaphragm and the pleura (20). 6) Comet-tail artifact: sonographic artifact with an appearance similar to that of the ring-down artifact but more attenuated, shorter, and tapering in depth as in the tail of a comet. The mechanism underlying comet tail artifact formation is reverberation (21). 7) Interstitial-alveolar syndrome (AIS): defined as the presence of more than 3 B-lines or the presence of areas of 'white lung' in every examined area (15). 8) Bilateral white lung: defined as the presence of compact B-lines in the 6 areas without horizontal reverberation (15). 9) Lung pulse: Lung sliding is replaced by a kind of pulsation, synchronized with heart activity, which is the early specific ultrasound sign of complete atelectasis (22).

\subsection{Statistical Analysis}

Data analyses were conducted using SPSS for Windows (Release 16.0, SPSS Inc, Chicago, IL). The ultrasound findings of the two groups were compared by Student's t test with 1 degree of freedom, and Fisher's exact test was used to confirm the results. We accepted $\mathrm{P}<0.01$ as indicating statistical significance.

\section{Results}

\subsection{General Information for Each Group}

The mean gestational age was $34.9 \pm 2.7$ weeks in RDS patients and $35.1 \pm 2.8$ weeks in controls. The mean birth weight was $2320 \pm 353 \mathrm{~g}$ in RDS neonates and $2297 \pm 411$ $\mathrm{g}$ in controls (Table 1 ). The average age of infants at the time of ultrasonography was $9.7 \pm 3.3$ hours after birth. The risk factors for RDS in term infants including selective cesarean section, severe birth asphyxia, maternalfetal infection and female sex are closely correlated with full-term neonatal RDS. As more than $96 \%$ of the patients in our NICU were transferred from other hospitals, and because of many other reasons (such as calling time, traffic conditions, long-distance transport, etc.), the interval between the onset of RDS and diagnosis was often more than 6-12 hours. Thus, in most (80\%) patients a severe grade (II//V) RDS was diagnosed.

\begin{tabular}{|c|c|c|c|c|c|}
\hline Groups & $\begin{array}{c}\text { Gender, } \\
\text { (male/female) }\end{array}$ & GA, w & Birth Weight, $g$ & CS/SL, $n$ & Grade in Chest X-Ray (cases) \\
\hline $\operatorname{RDS}(\mathbf{n}=\mathbf{5 0})$ & $32 / 28$ & $27^{+4}-41^{+3}$ & $1000-4120$ & $34 / 16$ grade II & 10 cases, grade III 20 cases, grade IV 20 cases \\
\hline Premature infants $(n=20)$ & $12 / 8$ & $27^{+4}-36^{+3}$ & 1000 and more & $13 / 7$ grade II & 8 cases, grade III 8 cases, grade IV 4 cases \\
\hline Term infants $(n=30)$ & $20 / 10$ & $37-41^{+3}$ & $1550-4120$ & 21/9 grade II & 2 cases, grade III 12 cases, grade IV 16 cases \\
\hline Controls $(\mathbf{n}=\mathbf{5 0})$ & $30 / 20$ & $26^{+2}-41^{+1}$ & $870-3990$ & $22 / 18$ & normal \\
\hline
\end{tabular}

\footnotetext{
a Abbreviations: CS, cesarean section; GA, gestational age; RDS, respiratory distress syndrome; SL, spontaneous labor.
} 
Liu Jet al.

\subsection{Normal Sonographic Lung Appearance}

The ultrasound appearance of normal lung is "black". The superficial layers of the thorax consist of subcutaneous tissue and muscles. On longitudinal scans, the ribs appear as curvilinear structures associated with posterior acoustic shadowing. The pleural line appears smooth, clear and shows a regular echogenic line with a width of less than $0.5 \mathrm{~mm}$. The presence of these artifacts is related to pathology and results from the fluid-rich subpleural interlobular septae, which are surrounded by air and identify AIS. The fetal lung is very rich in fluids and therefore B-lines can also be seen in healthy term newborns. In this group, B-lines were found in 12 healthy infants. The B-lines were not compact and rarely numerous, and were seen more often on the right side without a typical localization. AIS was seen in 8 healthy infants without any lung consolidation (Table 2 and Figure 1 ).

\subsection{Ultrasound Results in RDS Patients}

Lung consolidation with air bronchograms was seen in every (100\%) RDS infant, which was mainly located in the subpleural area in mild RDS (grade II in chest x-ray) and air bronchograms sometimes, were not visible (Figure 2). However, in severe RDS (grade III IV in chest x-ray) the area of lung consolidation was observed expanding into other areas and even involving deep lung tissue. Additionally the air bronchograms became more evident in patients with severe RDS (Figures 3 -5). Bilateral white lung (or AIS) was also seen in $100 \%$ of RDS patients (Figure 6). An abnormal pleural line (thickened or blurred) was observed in $100 \%$ of RDS patients (Figures 2 - 6). Additionally, A-lines had disappeared in $100 \%$ of RDS patients (Figures 2 - 5). Pleural effusion, which can co-exist with lung consolidation, was found in $8(16 \%)$ of the RDS patients (Figure 5). Lung pulse was seen in every grade III IV RDS patient (40 cases), while 10 grade II RDS patients did not show this sign. The extent and nature of lesions in bilateral lung were inconsistent. For example, one patient with $\mathrm{G}_{1} \mathrm{P}_{1}$, with a gestational age of $37^{+3}$ weeks and birth weight of $3100 \mathrm{~g}$, was delivered by cesarean section and admitted to our NICU due to difficulty in breathing. Arterial blood analysis showed $\mathrm{pH}$ of 6.91, $\mathrm{PaCO}_{2} 97 \mathrm{mmHg}, \mathrm{PaO}_{2} 25$ $\mathrm{mmHg}$, and BE-15.5 mmol/L. A chest $\mathrm{x}$-ray indicated grade
IV RDS. In this case, a lung ultrasound revealed a significant area of consolidation with air bronchograms in the left lung, with mild consolidation and a large amount of pleural effusion in the right lung (Figure 5).

\subsection{The Sensitivity and Specificity of Ultrasound Signs for the Diagnosis of Neonatal RDS}

Lung consolidation, pleural line abnormalities, bilateral white lung and A-lines disappearance were always seen in RDS patients and these signs have never been observed in controls. In our experience the simultaneous co-existence of lung consolidation, pleural line abnormalities and bilateral white lung, or lung consolidation, pleural line abnormalities and A-lines disappearance occurs with a sensitivity $(\mathrm{a} / \mathrm{a}+\mathrm{c})$ and specificity $(\mathrm{d} / \mathrm{b}+\mathrm{d})$ of $100 \%$ (Tables 3 and 4 ). Besides, lung pulse found by real-time ultrasound has the value of confirming RDS diagnosis. It can be seen from data that the sensitivity was $80 \%$ and specificity $100 \%$ of lung pulse for the diagnosis of neonatal RDS.

Figure 1. Normal Lung Ultrasound Appearance: Gestational Age $73^{+5}$ Weeks, Birth Weight $2570 \mathrm{~g}$

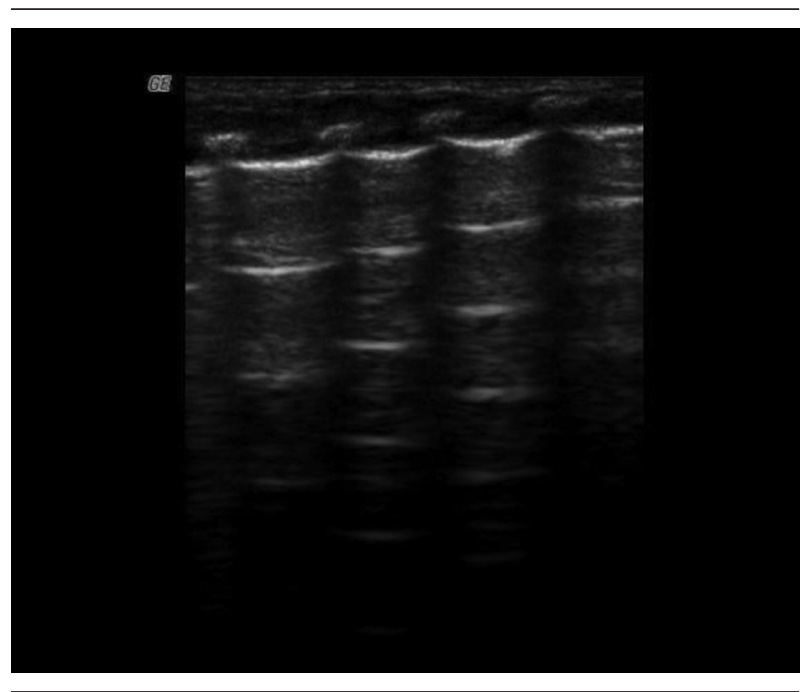

Longitudinal scan of the thorax, noting the 'black' normal lung and the echogenic line of normal pleura, paralleled A-lines and curvilinear appearance of the ribs associated with posterior acoustic shadowing. B-lines cannot be found in this infant.

Table 2. Distribution of Ultrasound Findings in the Two Groups ${ }^{a}$

\begin{tabular}{lccc}
\hline Ultrasound Findings & RDS Group $(\mathbf{n}=\mathbf{5 0})$ & Control Group $(\mathbf{n}=\mathbf{5 0})$ & P Value \\
\hline Consolidations & 50 & 0 & $<0.001$ \\
Pleural-line abnormalities & 50 & 0 & $<0.001$ \\
Bilateral "white lung" & 50 & 0 & $<0.001$ \\
A-lines & 0 & 50 & $<0.001$ \\
B-lines & 0 & 12 & $<0.001$ \\
Pleural effusion & 8 & 0 & $<0.001$ \\
Lung pulse & 40 & 0 & $<0.001$ \\
AIS & 50 & 8 & $<0.001$ \\
\hline
\end{tabular}

\footnotetext{
a Abbreviations: AIS, interstitial-alveolar syndrome; RDS, respiratory distress syndrome.
} 

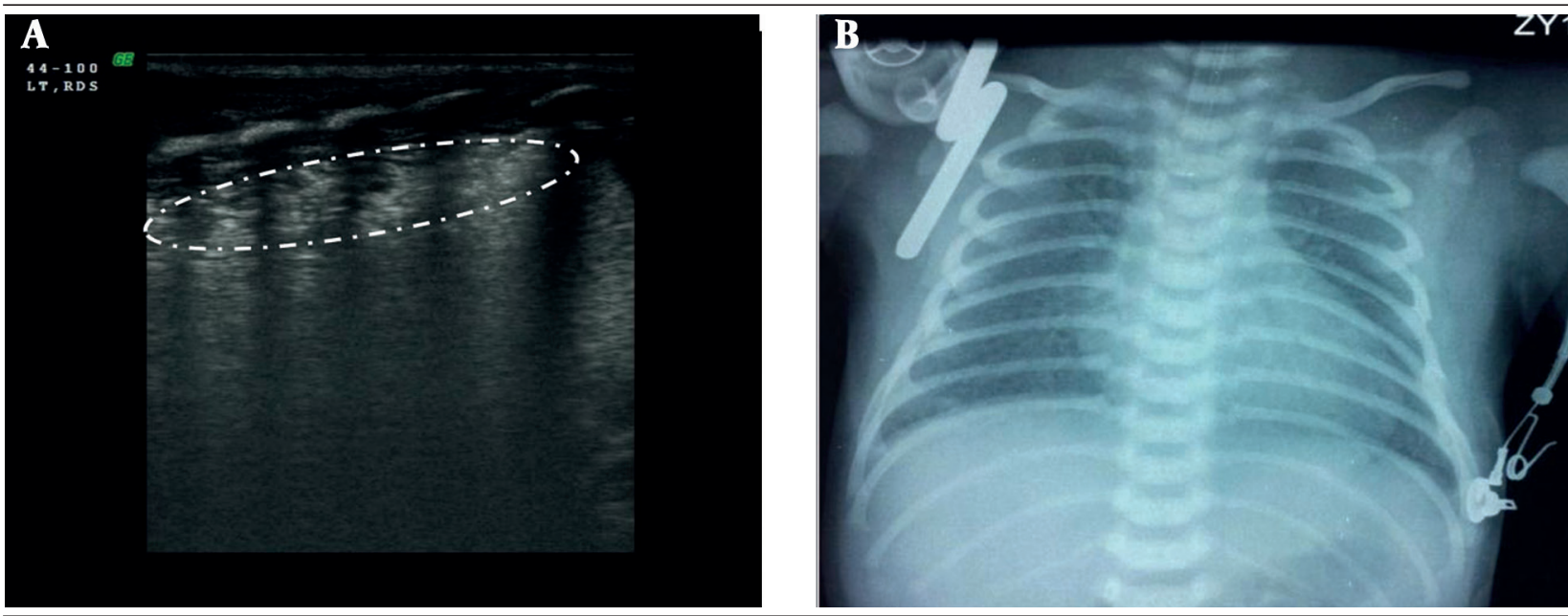

Figure 2. Grade II RDS

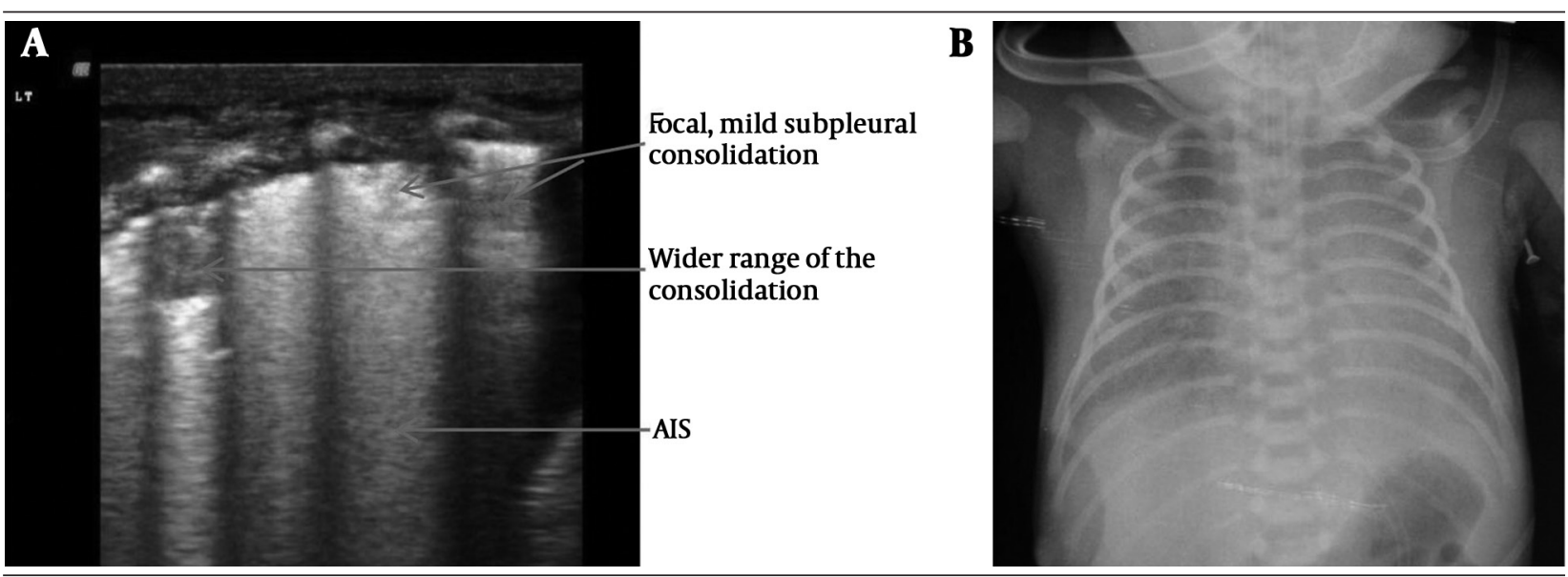

Figure 3. Grade III RDS
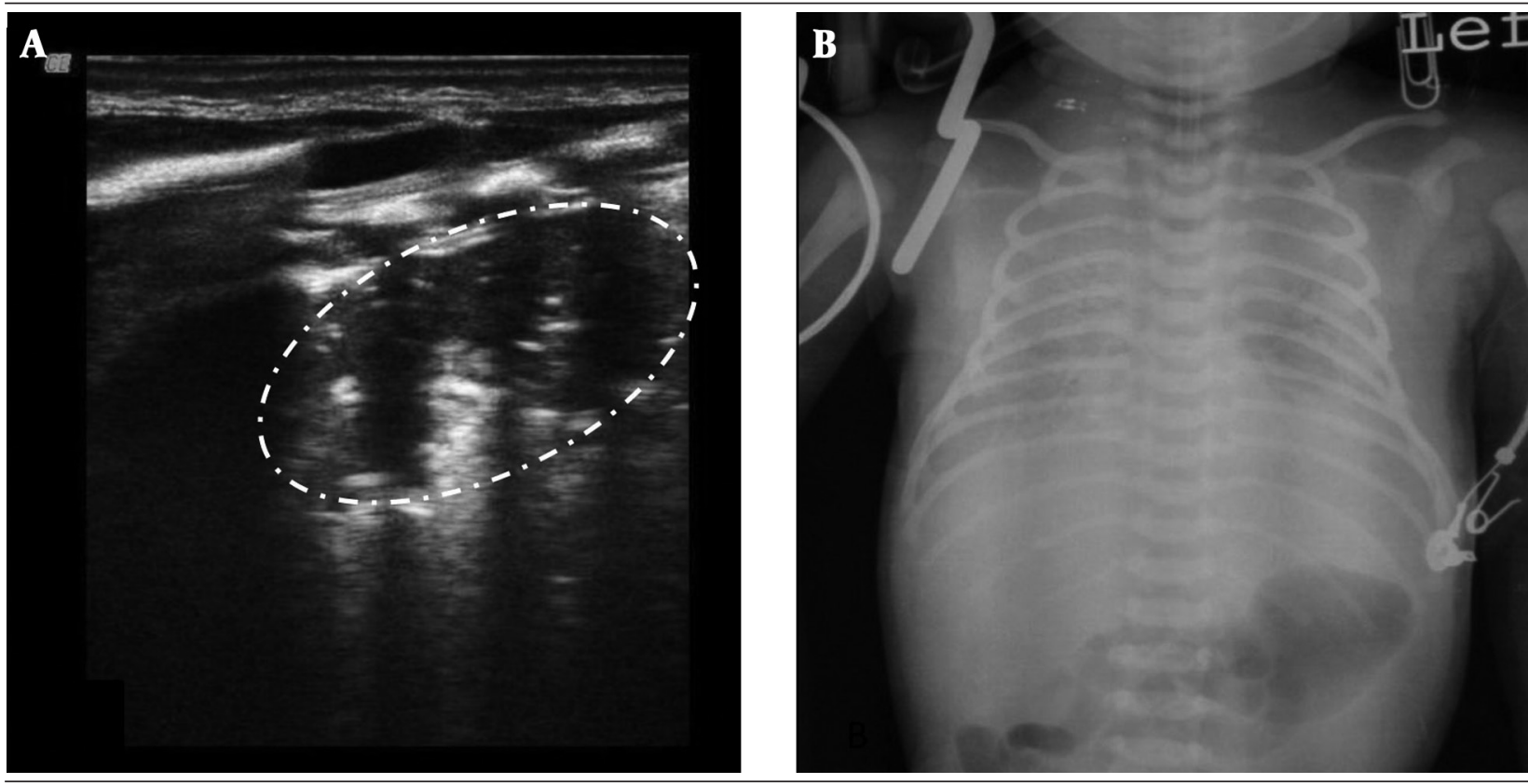

Figure 4. Grade IV RDS 

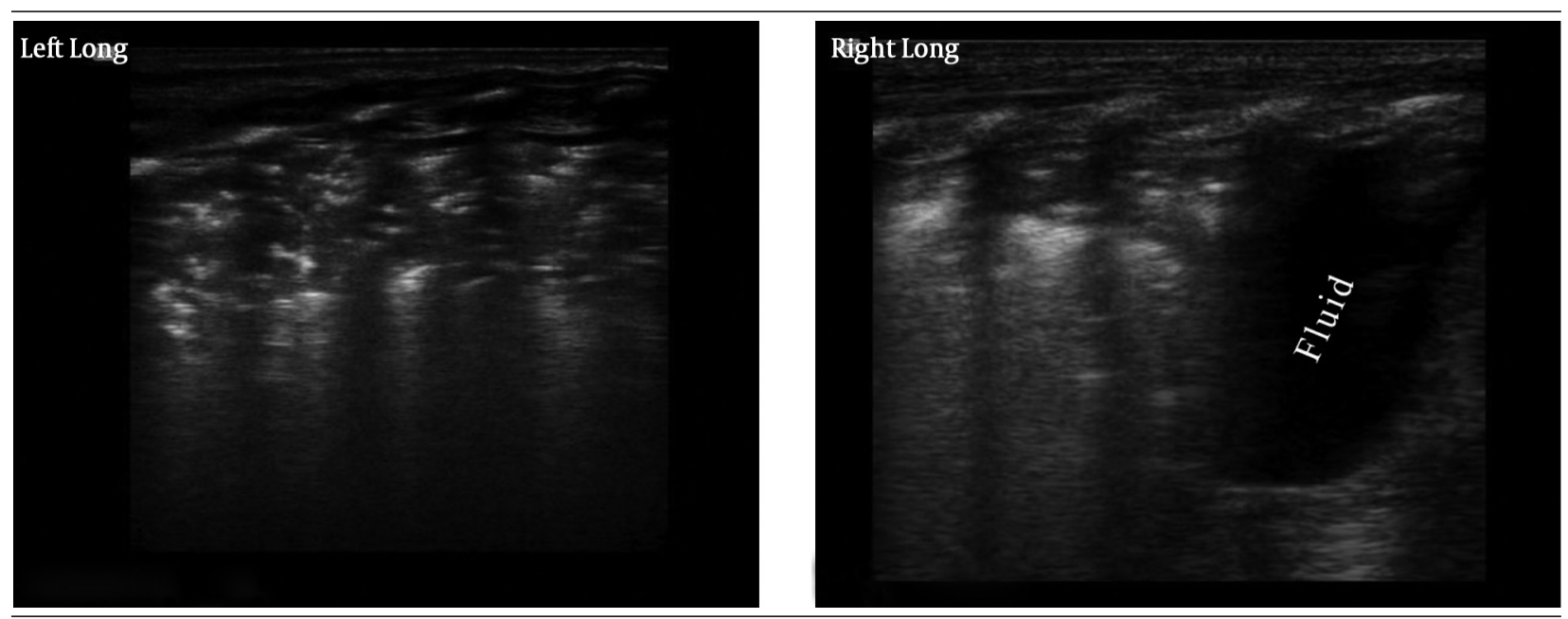

Figure 5. Different Ultrasound Appearance of Grade IV RDS Bilaterally in Lungs

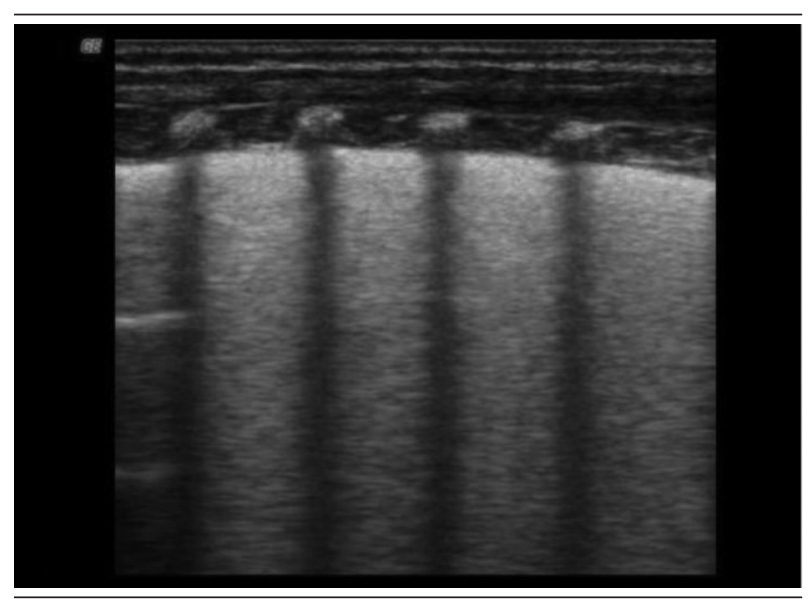

Figure 6. Ultrasound Image of White Lung (AIS)

Table 3. The Sensitivity and Specificity of Lung Consolidation, Pleural line Abnormalities and Bilateral White Lung for the Diagnosis of Neonatal RDS ${ }^{\text {a }}$

\begin{tabular}{lccc}
\hline & RDS & Control & Total \\
\hline With three signs & $50(\mathrm{a})$ & $0(\mathrm{~b})$ & $50(\mathrm{a}+\mathrm{b})$ \\
Without three signs & $0(\mathrm{c})$ & $50(\mathrm{~d})$ & $50(\mathrm{c}+\mathrm{d})$ \\
Total & $50(\mathrm{a}+\mathrm{c})$ & $50(\mathrm{~b}+\mathrm{d})$ & 100 \\
\hline
\end{tabular}

a Abbreviations: RDS, respiratory distress syndrome.

Table 4. The Sensitivity and Specificity of Lung Consolidation, Pleural Line Abnormalities and A-line Disappearance for the Diagnosis of Neonatal RDS ${ }^{\text {a }}$

\begin{tabular}{lccc}
\hline & RDS & Control & Total \\
\hline With three signs & $50(\mathrm{a})$ & $0(\mathrm{~b})$ & $50(\mathrm{a}+\mathrm{b})$ \\
Without three signs & $0(\mathrm{c})$ & $50(\mathrm{~d})$ & $50(\mathrm{c}+\mathrm{d})$ \\
Total & $50(\mathrm{a}+\mathrm{c})$ & $50(\mathrm{~b}+\mathrm{d})$ & 100 \\
\hline
\end{tabular}

a Abbreviations: RDS, respiratory distress syndrome.

\section{Discussion}

The results of this study confirm that lung ultrasonography has a very high sensitivity and specificity in the diagnosis of neonatal RDS. According to the findings of this study, the main features of RDS that can be visualized by ultrasound imaging include lung consolidation with air bronchograms, pleural line abnormalities, pleural effusion, lung pulse and bilateral white lung or alveolar-interstitial syndrome. The most important indicator of RDS in a lung ultrasound is lung consolidation, which can be seen in all RDS patients, but the extent and scope of the consolidation varies with the grade of RDS. Consolidation in grade II RDS may be limited to the subpleural, presents as focal and small-scale, and air bronchograms may not be visible. In contrast, the area of consolidation appears significantly expanded in severe RDS (grade III IV by chest x-ray), with air bronchograms becoming more obvious. Pleural line abnormality is one of the most common ultrasound observations in RDS patients, but also exists in other lung diseases such as pneumonia, bleeding, and transient tachypnea of the newborn (TTN). Therefore, pleural line abnormalities as visualized by ultrasound are not specific to RDS (23). According to the literature, the sensitivity and specificity of pleural line abnormalities for diagnosis of RDS are $100 \%$ and $45 \%$, respectively $(15,20)$. A-lines disappearance was found in $100 \%$ of RDS patients. This suggests that A-line disappearance is also a sonographic indicator of neonatal RDS. However, like pleural line abnormalities, A-line disappearance can also be found in other lung diseases. Therefore, it is not a change specific to RDS either (23). In our experience the simultaneous co-existence of lung consolidation, pleural line abnormalities and bilateral white lung, or lung consolidation, pleural line abnormalities and A-lines disappearance, occurs with a sensitivity and specificity of $100 \%$.

Traditionally, RDS is characterized by atelectasis, a severe RDS even appears only as "white lung" on a chest radiograph. This technique is incapable of visualizing pleural effusion, pulmonary edema or other pathologi- 
cal changes. Therefore, the results of lung ultrasound in these patients are completely changing our traditional concepts. First, RDS can exist not only with atelectasis, but also with pulmonary edema or pleural effusion. Second, the extent and nature of lesions in bilateral lung can be inconsistent (Figure 5). These findings suggest that a lung ultrasound can provide us with additional medical and clinical information. Because pulmonary edema and pleural effusion are also the main pathological features of TTN, "white lung" (AIS) or pleural effusion is not a specific symptom of RDS, as it is also found in TTN and other lung diseases $(16,20)$. According to the literature, the sensitivity and specificity of pleural effusion for diagnosing RDS are $66.6 \%$ and $5 \%$, respectively; the sensitivity and specificity of bilateral white lung or AIS for the diagnosis of RDS are $100 \%$ and $0 \%$, respectively (15).

According to the findings of this study, lung pulse is one of the most important features of RDS that can be visualized by ultrasound imaging. Lung pulse was found in all (40 cases, $100 \%$ ) grade III IV RDS infants, while it was not seen in the remaining 10 patients with grade II RDS. Thus we believe that lung pulse is associated with the degree and the extent of lung consolidation. According to our results the sensitivity was $80 \%$ and specificity $100 \%$ of lung pulse for the diagnosis of neonatal RDS.

In short, this study further confirms that a lung ultrasound has great significance in diagnosing neonatal RDS. As indicated by Copetti et al. (15), the lung ultrasound has many advantages. First, being non-ionizing, the ultrasound can be performed at the bedside. Second, the ultrasound is easy to operate, and can be repeated several times a day without hazards to the operator or the patient. Third, this is a low-cost technique, which requires only elementary skills. Last but not least, the lung ultrasound has very high accuracy and reliability in diagnosing pulmonary disease. Given all of these, in certain advanced neonatal intensive care units this method could replace radiography as the first-line diagnostic approach in neonatal period (24). However, there are some limitations in this study worth noting. The number of RDS infants was limited, and some acute complications secondary to air leak syndrome (such as pneumomediastinum, interstitial emphysema, pneumopericardium) cannot easily be discovered by using ultrasound. According to the results of this study, maybe ultrasound is not proper for the diagnosis of grade I RDS. More research is needed to replace chest $\mathrm{x}$-ray by sonography in follow-up scanning of RDS after treatment.

\section{Acknowledgements}

We thank Miss Gao Guang-Pei from Chinese Union Medical University, who have polished this paper carefully.

\section{Funding/Support}

This work was supported by China Postdoctoral Science Foundation (No. 20080431405 \& No. 200801041).

\section{References}

1. Koivisto M, Marttila R, Kurkinen-Raty M, Saarela T, Pokela ML, Jouppila P, Hallman M, et al. Changing incidence and outcome of infants with respiratory distress syndrome in the 1990s: a population-based survey. Acta Paediatr. 2004;93(2):177-84.

2. Ayachi A, Rigourd V, Kieffer F, Dommergues MA, Voyer M, Magny JF. [Hyaline membrane disease in full-term neonates]. Arch Pediatr. 2005;12(2):156-9.

3. Bouziri A, Ben Slima S, Hamdi A, Menif K, Belhadj S, Khaldi A, Kechaou W, Kazdaghli K, Ben Jaballah N, et al. [Acute respiratory distress syndrome in infants at term and near term about 23 cases]. Tunis Med. 2007;85(10):874-9.

4. Faix RG, Viscardi RM, DiPietro MA, Nicks JJ. Adult respiratory distress syndrome in full-term newborns. Pediatrics. 1989;83(6):971-6.

5. Liu J, Shi Y, Dong JY, Zheng T, Li JY, Lu LL, Liu JJ, Liang J, Zhang H, Feng ZC, et al. Clinical characteristics, diagnosis and management of respiratory distress syndrome in full-term neonates. Chin Med J (Engl). 2010;123(19):2640-4.

6. Zechner PM, Seibel A, Aichinger G, Steigerwald M, Dorr K, Scheiermann P, Schellhaas S, Cuca C, Breitkreutz R, Arbeitsgruppe des Moduls 5 in Anasthesie Fokussierte Sonographie der D, et al [Lung ultrasound in acute and critical care medicine]. Anaesthesist. 2012;61(7):608-17.

7. Reissig A, Gramegna A, Aliberti S. The role of lung ultrasound in the diagnosis and follow-up of community-acquired pneumonia. EurJ Intern Med. 2012;23(5):391-7.

8. Dexheimer Neto FL, Dalcin Pde T, Teixeira C, Beltrami FG. Lung ultrasound in critically ill patients: a new diagnostic tool. J Bras Pneumol. 2012;38(2):246-56.

9. Caiulo VA, Gargani L, Caiulo S, Fisicaro A, Moramarco F, Latini G, Picano E, et al. Lung ultrasound in bronchiolitis: comparison with chest X-ray. Eur J Pediatr. 2011;170(11):1427-33.

10. Santuz P, Bonetti P, Serra A, Biban P. Ultrasound-guided lung recruitment in a young infant with ARDS. Paediatr Anaesth. 2010;20(9):895-6.

11. Barillari A, Fioretti M. Lung ultrasound: a new tool for the emergency physician. Intern Emerg Med. 2010;5(4):335-40.

12. Colmenero M, Garcia-Delgado M, Navarrete I, Lopez-Milena G. [Utility of the lung ultrasound in the intensive medicine unit]. Med Intensiva. 2010;34(9):620-8.

13. Elia F, Verhovez A, Molino P, Ferrari G, Apra F. Lung ultrasound in the reexpansion of pulmonary atelectasis. Intern Emerg Med. 2011;6(5):461-3.

14. Bober K, Swietlinski J. Diagnostic utility of ultrasonography for respiratory distress syndrome in neonates. Med Sci Monit. 2006;12(10):CR440-6.

15. Copetti R, Cattarossi L, Macagno F, Violino M, Furlan R. Lung ultrasound in respiratory distress syndrome: a useful tool for early diagnosis. Neonatology. 2008;94(1):52-9.

16. Copetti R, Cattarossi L. The 'double lung point': an ultrasound sign diagnostic of transient tachypnea of the newborn. Neonatology. 2007;91(3):203-9.

17. Lichtenstein D, Meziere G, Biderman P, Gepner A. The comet-tail artifact: an ultrasound sign ruling out pneumothorax. Intensive Care Med.1999;25(4):383-8.

18. Lichtenstein DA, Lascols N, Meziere G, Gepner A. Ultrasound diag nosis of alveolar consolidation in the critically ill. Intensive Care Med. 2004;30(2):276-81.

19. Lichtenstein D, Meziere G, Biderman P, Gepner A, Barre O. The comet-tail artifact. An ultrasound sign of alveolar-interstitial syndrome. Am J Respir Crit Care Med.1997;156(5):1640-6.

20. Lichtenstein D. Lung ultrasound in the critically ill. Clin Intensive Care. 2005;16(2):79-87.

21. Soldati G, Copetti R, Sher S. Sonographic interstitial syndrome: the sound of lung water. JUltrasound Med. 2009;28(2):163-74.

22. Lichtenstein DA, Lascols N, Prin S, Meziere G. The "lung pulse": an early ultrasound sign of complete atelectasis. Intensive Care Med. 2003;29(12):2187-92.

23. Liu J. Lung ultrasonography for the diagnosis of neonatal lung disease. J Matern Fetal Neonatal Med. 2014;27(8):856-61.

24. Cattarossi L, Copetti R, Poskurica B. Radiation exposure early in life can be reduced by lung ultrasound. Chest. 2011;139(3):730-1. 